\title{
Investigation of the Adherence Influence on the Dynamic Behavior of the Vehicle
}

\author{
Nacer Hamadi ${ }^{1}$, Djamel-eddine Ameddah ${ }^{1}$ and Hocine Imine ${ }^{2}$ \\ ${ }^{1}$ Department of Electronic, Advanced Electronic Laboratory (A.E.L.) \\ Faculty of technologies, Batna University, Alegria \\ nacer.hamadi@univ-batna.dz,Abida_dj@live.fr \\ ${ }^{2}$ French institute of Sciences and Technologies of Transport, Installation and the \\ Networks, University Paris-East, LEPSIS, IFSTTAR, France \\ hocine.imine@ifsttar.fr \\ Abstract \\ In this paper, we were interested on the adherence influence on the dynamic behavior of the \\ vehicle. With this intention, we based ourselves on a grticulated nominal dynamic model of a \\ ground vehicle as well as the contact tire-road.
}

Keywords: vehicle; modeling; tire; road; adherence; simulation; Lagrange formalism

\section{Introduction}

A vehicle is a nonlinear complex ystem witk its knematics and dynamic characteristics (actuators, thermal engine); moreover, and particularly in all ground, it can be subjected to many disturbances, such as changes of adherence of the ground or slope, strong gales, etc.

In this paper, one will be interested in the study of the adherence influence on the dynamic behavior of the vehicle for the various types of lanes [1-3]. For this study, we used the structure defined by the diagram of the Figure 1 . The structure is based mainly on the direct dynamic model of the physical model more precise and realistic than the bicycle model or quarter model used usually for some control purposes developed in [7-12] and the entries such as the orders of the driver (flying, accelerating, brake), the aerodynamics effects and the road entries.

Indeed, the adberence influence represents the effects between the tire and the ground; these effects on the level of the contacts points enter in all the dynamic exchanges, which condition the handling of the vehicle. The major part of the efforts applied to the vehicle pass through the tires, which depend on the type and the carriageway surfacing.
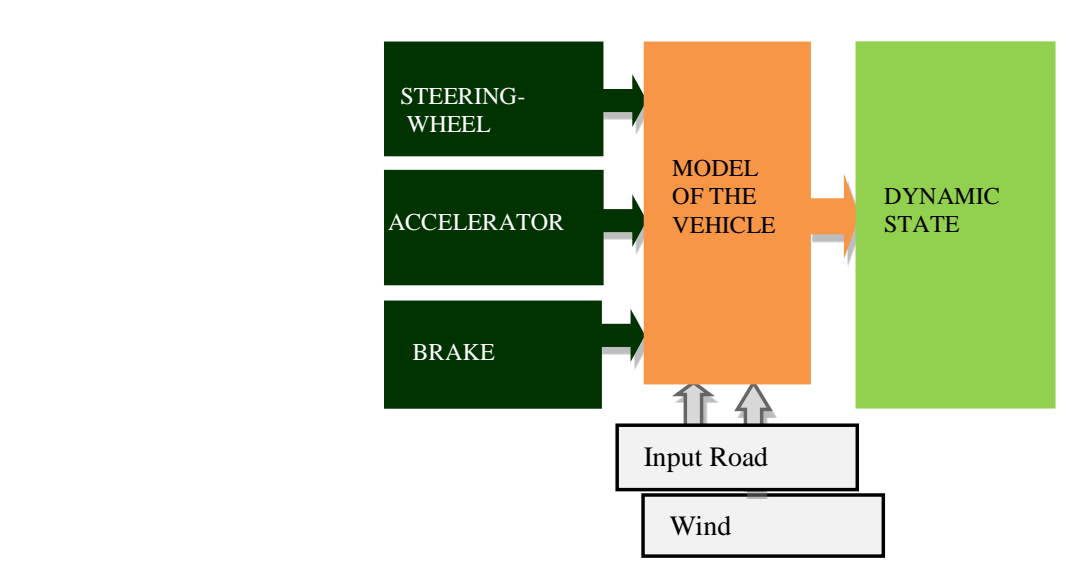

Figure 1. Diagram Simulator of the Vehicle 
In this paper we employed the empirical formula suggested by Bakker and Pacejka [13] to describe the behavior of the tire. We will concentrate on models who take account of the factors of environment (load, nature of the roadway) or of the intrinsic properties of the tire (structure of the tire, rigidity, pressure). To represent, the tire longitudinal and side behavior in the nonlinear case, the formula of Pacejka makes it possible to define by the same basic formula the longitudinal force, the lateral force and the self-aligning torque under conditions of pure sideslip or pure braking expressed in the pneumatic reference. The parameters on which these functions depend are sideslip, longitudinal slip, the camber angle and the vertical load. If we base on the results of a test carried out for tires coefficients Pacejka measured by ARIANE within the IFSTTAR (ex LCPC 1), for the dryness and wet lanes to calculate the longitudinal and side contacts forces.

This paper is organize as follows; In the second part, we present the modeling of the vehicle, in the third part, we discus and analyze the results of simulation, and an experimental validation is shown, in particular by the results of the influence of the adherence on the dynanic behavior of the vehicle. Finally, a conclusion and some prospects is given in the last part.

\section{Vehicle Modelling}

\subsection{Vehicle Frame}

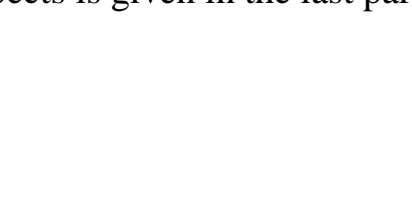

To calculate the operational dynamic model, smplifications related to the dynamic structure of the vehicle are essential:

- The chassis is regarded as a rigid $3 \mathrm{~d}$ body

- Each wheel is regarded as a rigíd disk and it is assumed to be represented by a single point of contact with the terrain surface.

- The steering of the direct wheels, clearances of the suspensions and the rotation of the wheels can represent the kinematics of connection tire/road.

The Figure 2 illustrates all the kinemalics joints considered in the used model.

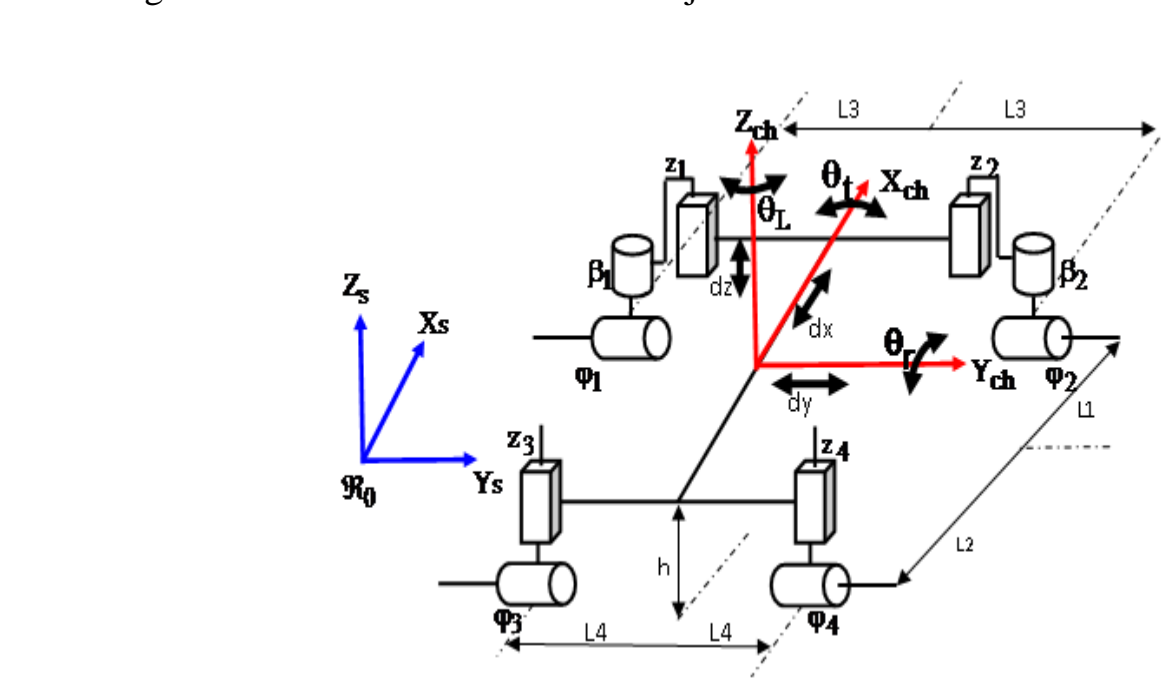

Figure 2. Coordinate System to described the Vehicle Motion

The nominal dynamic model considered with 16 DoF to divide like following:

- The Frame with 6 DoF accounts of three translator movements (x, y, z) and three 
rotational movements rolling-pitching-yaw $\theta_{r}, \theta_{t}, \theta_{l}$ according to three axes' $\mathrm{x} \mathrm{y} \mathrm{z}$ The suspension with 4 DoF represents 4 variables of clearances $(z 1, z 2, z 3, z 4)$.

- The direction with $2 \mathrm{DoF}$ respectively represents the steering angles left and right of the front steer wheels $\left(\beta_{1}, \beta_{2}\right)$.

- The wheels with 4 DoF represents the rotation angles of the four wheels around the fused axis $\left(\varphi_{1}, \varphi_{2}, \varphi_{3}, \varphi_{4}\right)$.

The generalized coordinate's variable vector $\mathrm{q} \in \mathbb{R}^{16}$ defined by:

$$
q^{T}=\left[x, y, z, \theta_{r}, \theta_{t}, \theta_{l}, z 1,, z 2, z 3, z 4, \beta_{1}, \beta_{2}, \varphi_{1}, \varphi_{2}, \varphi_{3}, \varphi_{4}\right]
$$

The dynamic parameters considered are the mass of the chassis MS, the mass of each front wheel MRF, the mass of each rear wheel MRR, and h1and h2 is the initial length spring of the front suspension and $\mathrm{h} 3$ and $\mathrm{h} 4$ is the initial length spring of the rear suspension

\subsection{Dynamic Model}

The dynamic model of the vehicle is obtained by using the approach of Lagrange based on the calculation of the kinetic and potential energy of the complete system. The motion equation of the mechanism is given by:

$$
M(q) \ddot{q}+C(q, \dot{q} q+G(q)=
$$

$\Gamma=\tau_{m}+\tau_{D}-\tau_{r r}-\tau_{\text {susp }}+\Gamma_{a}$ is the vector of the internal and external forces between the vehicle bodies. Where $\tau_{m}$ represent the engine forque of the two driving wheels, direction torque of the two cap wheels is $\tau_{D}$, roling friction torque of the 4 wheels is $\tau_{r r}$, torques of the 4 suspensions is $\tau_{\text {susp }}$ and $\Gamma_{a}$ is the vector of contact forces on each wheel (see section 3 ).

$M q \in \mathbb{R}^{16 \times 16}$ Represent the matrix of the kinetic energy, named the inertia system matrix. It is symmetrical, definite and positive. Of these elements, are functions of the joint variables $\mathrm{q}_{\mathrm{i}}$. The total kinetic energy is calculated by the formula:

$$
\text { Q } T=\frac{1}{2} \dot{q}^{T} M(q) \dot{q}
$$

And $\mathrm{M}(q)$ is given by $: 0$

\section{With}

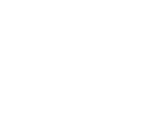

$$
M(q)=\sum_{i=1}^{5} m i J_{v_{i}}^{T}(q) J_{v_{i}}(q)+J_{w_{i}}^{T}(q) R_{i}(q) I_{i} R_{i}^{T}(q) J_{w_{i}}
$$

\footnotetext{
$J(4)$ Jacobian matrix transposed of the linear velocity for body i,

$J_{w_{i}}^{T}(q)$ Jacobian matrix transposed of the angular velocity for body i,

$R_{i}(q)$ Rotate matrix of body i,

$I_{i} \quad$ Inertia matrix of body $\mathrm{i}$,

$m_{i} \quad$ Mass of body $i$.
} 
To calculate the Jacobian matrix $\left(J_{v_{i}}^{T}(q)\right.$ and $\left.J_{w_{i}}^{T}(q)\right)$ ?and rotation matrix $R_{i}(q)$, we chose, the homogeneous matrix based on the translation and rotation, who define the movement of a body in relation to another, the homogeneous matrix is given by:

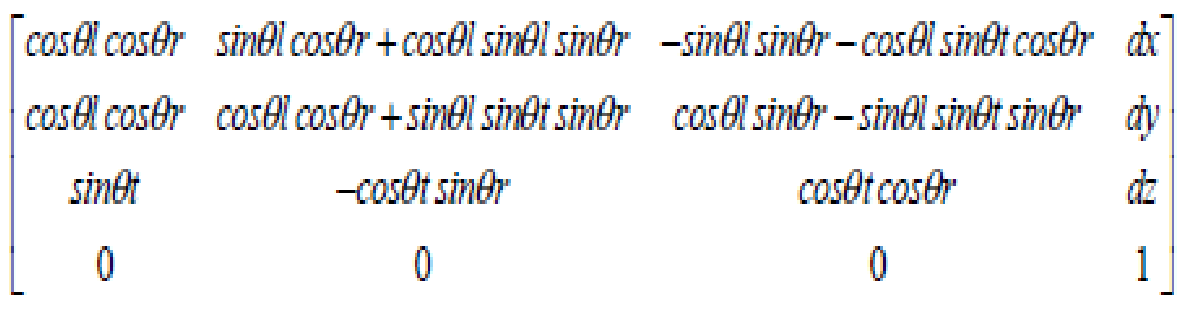

$C(q, \dot{q}) \in \mathrm{R}^{16 \times 16 ?}$ Represent the matrix of the Coriolis and Centrifuges terms. It is of dimension (16x16). The calculation of the dynamic coefficients of the matrix $C(q, \dot{q})$ is established by respecting the property of passivity of the system. A way of calculating the coefficients of the matrix is to use the symbols of Christopher.

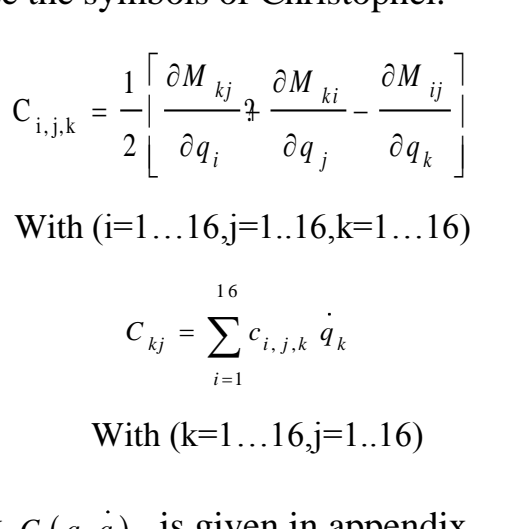

The form symbolic of the matrix $C(q, q)$ is given in appendix.

$G(q)$ is the vector of the gravity forces; It given by the gradient of the potential energy EP versus to $q$, The potential energy of the $\mathrm{i}$-th body can be computed by assuming that the mass of the entire object is concentrated at its center of mass and is given by

$$
E P_{i}=g^{T} r_{c i} m_{i}
$$

Where gis/vector giving the direction of gravity in the inertial frame and the vector $r_{c i}$ gives the coordinates of the center of mass of body $i$. The total potential energy of the 5-body of our system is therefore

$$
E P=-g(M S . Z-M R F(h 1+h 2-Z 1-Z 2-2 Z)-M R R .(h 3+h 4-Z 3-Z 4-2 Z))
$$

So

$$
\begin{aligned}
G(q)=[0,0,-g \cdot(M S & +2 \cdot M R F+2 M R R), 0,0,0, \\
& -g M R F,-g \cdot M R F,-g M R R,-g \cdot M R R, 0,0,0,0,0,0]^{T}
\end{aligned}
$$


Finally, the 16 DoF nominal model proposed is then equivalent to:

$$
\left[\begin{array}{ccc}
M_{1,1} & \cdots & M_{1,16} \\
\vdots & \ddots & \vdots \\
M_{16,1} & \cdots & M_{16,16}
\end{array}\right]\left[\begin{array}{c}
\dot{q}_{1} \\
\vdots \\
\ddot{q}_{16}
\end{array}\right]+\left[\begin{array}{ccc}
C_{1,1} & \cdots & C_{1,16} \\
\vdots & \ddots & \vdots \\
C_{16,1} & \cdots & C_{16,16}
\end{array}\right]\left[\begin{array}{c}
\dot{q}_{1} \\
\vdots \\
\dot{q}_{16}
\end{array}\right]+\left[\begin{array}{c}
G_{1} \\
\vdots \\
G_{16}
\end{array}\right]+\tau_{\text {susp }}+\tau_{r r}=\Gamma_{a}+\tau_{m}
$$

The resolution of the mechanism movements is equivalent to solving the following numerically equation $[5,6]$ :

$$
\ddot{q}=M^{-1}(q)[\Gamma-\mathrm{C}(\mathrm{q}, \dot{\mathrm{q}}) \dot{\mathrm{q}}-\mathrm{G}(\mathrm{q})]
$$

The equivalent state space representation,Let the state vector is

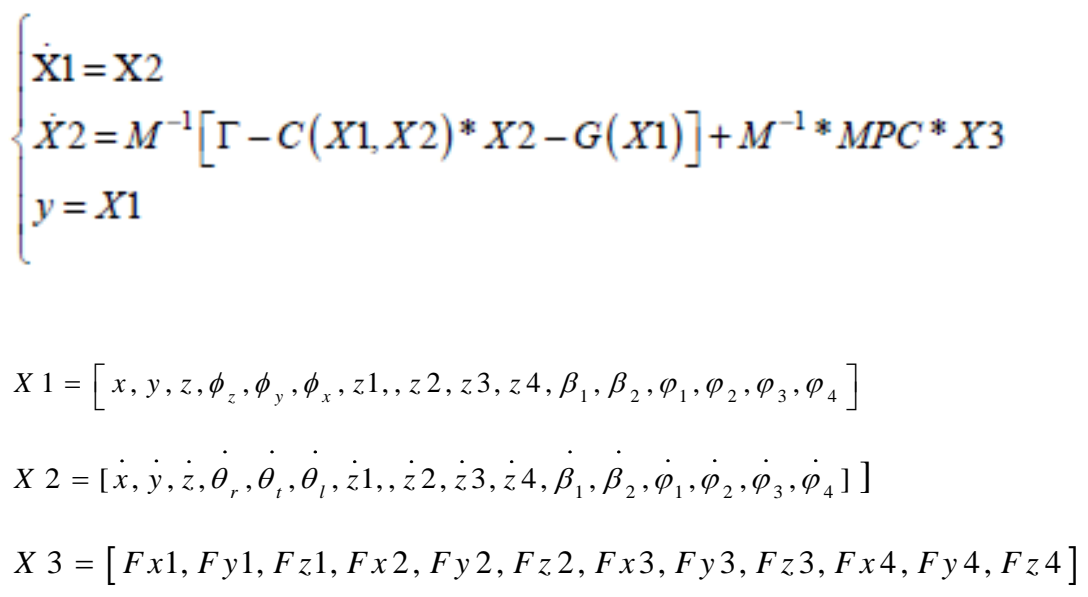

$\left.X 2=\left[\dot{x}, \dot{y}, \dot{z}, \dot{\theta}_{r}, \dot{\theta}_{t}, \dot{\theta}_{l}, \dot{z} 1,, \dot{z} 2 \dot{z} 3, \dot{z} 4, \dot{\beta}_{1}, \dot{\beta}_{2} \dot{\varphi}_{1}, \varphi_{2}, \dot{\varphi}_{3}, \dot{\varphi}_{4}\right]\right]$

$X 3=[F x 1, F y 1, F z 1, F x 2, F y 2, F z 2 F x 3, F y 3, F z 3, F x 4, F y 4, F z 4]$

$M(X 1) \in \mathbb{R}^{16 \times 16}$ Inertia matrix of the system is a symmetric positive definite

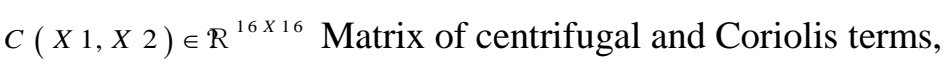

$G(X 1) \in \mathbb{R}^{16 X 1}$ is the Vector of gravity terms,

$M P C=J^{T} * M R$ Passage matrix of the 4 points of contacts relatively to the absolute reference, $J^{T} \in \mathbb{R}^{16 \times 12}$

$M R=\Re_{i}\left(\psi, \psi_{y}, \psi_{z}\right) \in \mathbb{R}^{12 X 12}$

road relatively to absolute reference,

$X 3 \in R^{12}$ represent the forces of the 4-point of contacts; the longitudinal, Side and vertical force, $\Gamma$ is the vector of the internal and external forces between the vehicle bodies. 


\subsection{Interaction Wheel-ground}

2.3.1. Dynamic Modelling of the Tire: In the case of pure slip, the general form of the basic model proposed to express the expression of the tangential forces is given in $([7,10,13])$ :

$$
\begin{gathered}
y(x)=D \sin \{C \arctan [B x-E(B x-\operatorname{rctan} B x)]\} \\
Y(x)=Y(X)+S v \\
x=X+S h
\end{gathered}
$$

The curve obtained by the above equation is represented in the Figure 3, whose pace makes it possible to find easily great number of these parameters:

B: factor of stiffness

C: factor of form

D: value of peak (compared to axis $\mathrm{X})(\mathrm{N})$

E: factor of curve

BCD: rigidity of drift (slope in the beginning)

Sv, Sx: offset vertical/longitudinal

$\mathrm{x}$ : drift angle (rad)/slip (\%)

$\mathrm{y}$ : variable of output (Fx or Fy) (N)

Like bases of parameter setting of the tires, we have the curves of the following [LCPC] adherences (longitudinal and side) Figure 4:

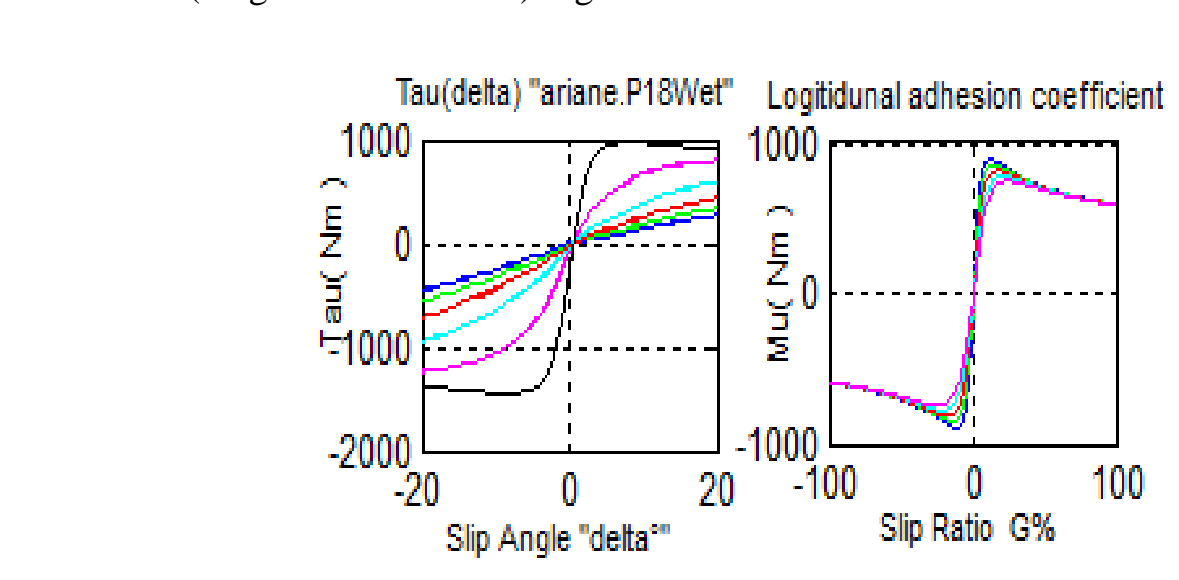

Figure 4. Relationships between Longitudinal and Side Adhesion Coefficient using the Magic Formula

2.3.2. Forces of Tire/Road Contact: The efforts at each contact tire/road are, the longitudinal 
force $F_{0 x i}$, the side force $F_{0 y i}$ and the normal force $F_{0 z i}$ Figure 2.

$$
F_{0 i}=\left[F_{0 x i}, F_{0 y i}, F_{0 z i}\right]^{T}
$$

For the case of the four-wheeled vehicle, the action of the forces of contact on each wheel is expressed by:

$$
\Gamma_{a}=\sum_{i=1}^{4} \Gamma_{a i}=J^{T} F_{0}
$$

With: $F_{0} \in \mathfrak{R}^{12}$ is the vector of the contact forces and $J^{T} \in \mathfrak{R}^{12 \times 15}$ (see appendix) is the matrix such as:

$$
\begin{gathered}
J^{T}=\left[\begin{array}{llll}
J_{1}^{T} & J_{2}^{T} & J_{3}^{T} & J_{4}^{T}
\end{array}\right] \\
F_{0}=\left[\begin{array}{llll}
F_{01}^{T} & F_{02}^{T} & F_{03} & F_{04}^{I}
\end{array}\right]
\end{gathered}
$$

If the plan of the surface of contact wheel ground forms an angle $\psi_{x}$ at axis $\overline{\mathrm{Ox}}_{0}$ an angle $\psi_{y}$ at axis $\overrightarrow{O y_{0}}$ and an angle $\psi_{z}$ at axis $\overline{Q z}$ then one has:

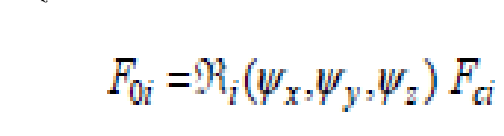

$$
\text { Q) }
$$

With $\mathfrak{R}_{i}\left(\psi_{x}, \psi_{y}, \psi_{z}\right)$ is the matrix of rotation of the reference frame of the wheel $\mathrm{i}$ whose origin is fixed at the center of the zone of contact, towards the absolute reference frame.

$$
\begin{aligned}
& \text { - Steerable wheels } \\
& \mathrm{R}_{i=1,2}\left(\psi_{x}, \psi_{y} \psi_{z}\right)= \\
& {\left[\begin{array}{cc}
\cos \left(\psi_{z}+\beta b \cos \left(\psi_{y}\right)\right. & \sin \left(\psi_{z}+\beta b\right) \cos \left(\psi_{x}\right)+\sin \left(\psi_{y}\right) \sin \left(\psi_{x}\right) \cos \left(\psi_{z}+\beta b\right) \\
\sin \left(\psi_{z}+\beta b\right) \cos \left(\psi_{x}\right) & \cos \left(\psi_{z}+\beta b\right) \cos \left(\psi_{x}\right)+\sin \left(\psi_{y}\right) \sin \left(\psi_{x}\right) \sin \left(\psi_{z}+\beta b\right) \\
\sin \left(\psi_{y}\right) & -\cos \left(\psi_{y}\right) \sin \left(\psi_{x}\right) \\
-\sin \left(\psi_{z}+\beta b\right) \sin \left(\psi_{x}\right)-\sin \left(\psi_{y}\right) \cos \left(\psi_{x}\right) \cos \left(\psi_{z}+\beta b\right) \\
\cos \left(\psi_{z}+\beta b\right) \sin \left(\psi_{x}\right)-\sin \left(\psi_{y}\right) \cos \left(\psi_{x}\right) \cos \left(\psi_{z}+\beta b\right)
\end{array}\right]}
\end{aligned}
$$

- No-Steer wheels 


$$
\begin{array}{r}
\mathrm{R}_{i=3,4}\left(\psi_{x}, \psi_{y} \psi_{z}\right)= \\
\left\{\cos \left(\psi_{z}\right) \cos \left(\psi_{y}\right)\right. \\
\sin \left(\psi_{z}\right) \cos \left(\psi_{y}\right) \\
\sin \left(\psi_{y}\right)
\end{array}
$$$$
\begin{aligned}
& \sin \left(\psi_{z}\right) \cos \left(\psi_{x}\right)+\cos \left(\psi_{z}\right) \sin \left(\psi_{y}\right) \sin \left(\psi_{x}\right) \\
& \cos \left(\psi_{z}\right) \cos \left(\psi_{x}\right)+\sin \left(\psi_{z}\right) \sin \left(\psi_{y}\right) \sin \left(\psi_{x}\right) \\
& -\cos \left(\psi_{y}\right) \sin \left(\psi_{x}\right)
\end{aligned}
$$$$
\left.-\sin \left(\psi_{z}\right) \sin \left(\psi_{x}\right)-\cos \left(\psi_{z}\right) \sin \left(\psi_{y}\right) \sin \left(\psi_{x}\right)\right\rceil
$$$$
\cos \left(\psi_{z}\right) \sin \left(\psi_{x}\right)-\sin \left(\psi_{z}\right) \sin \left(\psi_{y}\right) \cos \left(\psi_{x}\right)
$$$$
\cos \left(\psi_{y}\right) \cos \left(\psi_{x}\right)
$$

2.3.3. The Surface Plan of Tire / Road Contact: The road is defined generally by angles of Rolling-Pitching-Lace $\left(\psi_{x}, \psi_{y}, \psi_{z}\right)$ in space to the absolute reference frame. These angles are choosing as represented in the (Figure 5).

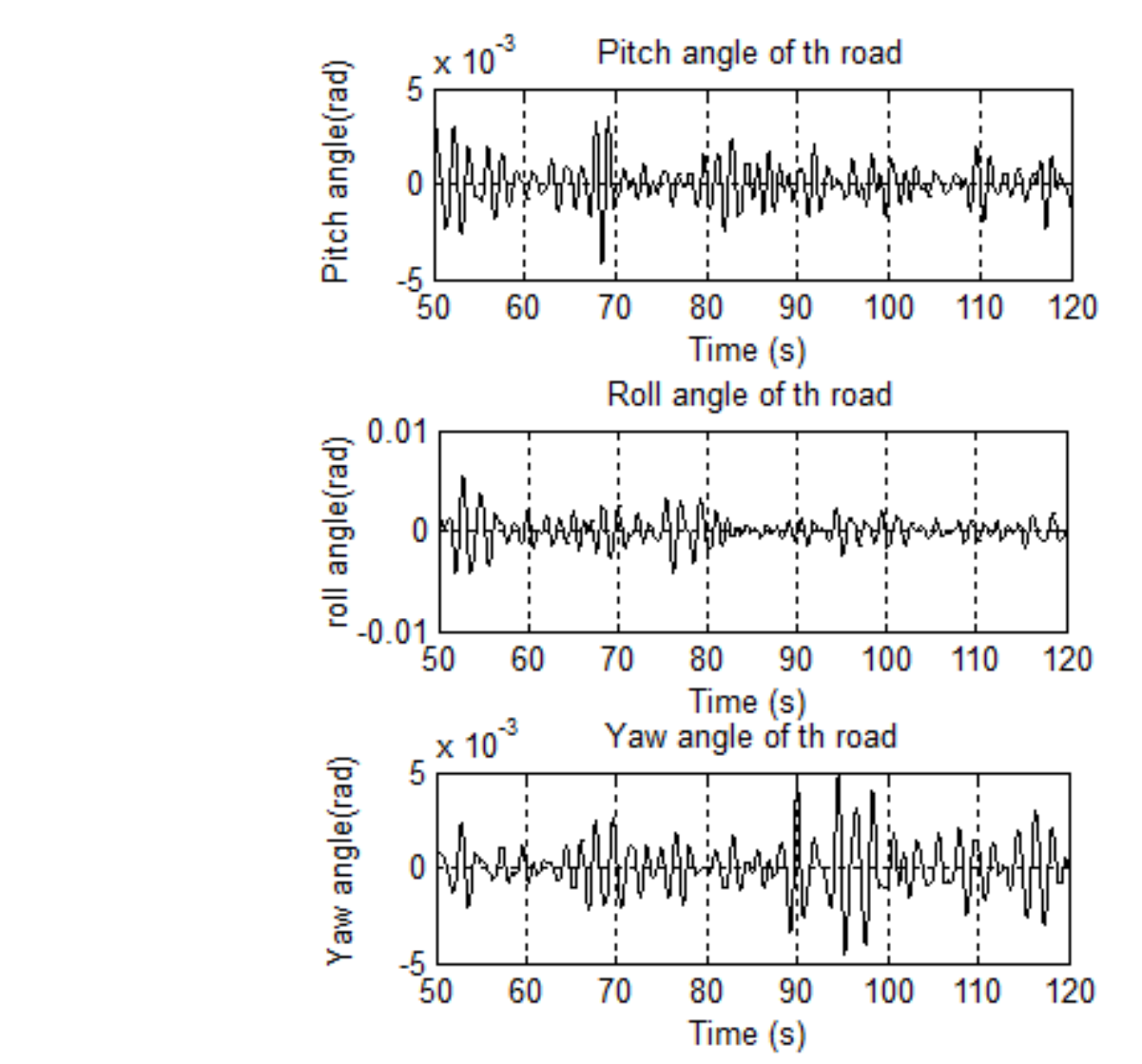

Figure 5. Plan Angles of the tire/road Surface Contact

2.3.4. Longitudinal Slip: The longitudinal slip $\mathrm{G}$ or a wheel $\mathrm{i}$ is given by the following expression [4] [7]: 


$$
\begin{cases}G_{i}=\frac{R w_{i}}{V_{x i}}-1 & \text { if } V_{x i}>R w_{i} \\ G_{i}=1-\frac{R w_{i}}{V_{x i}} & \text { if } R w_{i}<V_{x i}\end{cases}
$$

$V_{x i}$ Is the speed of the wheel center; $\mathrm{R}$ is the effective ray of the wheel: when a vertical force as applied to the tire, this one undergoes a crushing, which reduces the ray of the wheel

2.3.5. Sideslip Angle: The mathematical formulas of the sideslip angles, expressed in the reference frame related to the vehicle is given by the system (16) respectively, for the wheels: front-left, front-right, rear-left and rear-right [7].

With:

$\alpha_{\mathrm{i}} \quad$ Sideslip angle of the tire

$V_{y} \quad$ Lateral velocity of the vehicle.

$V_{x} \quad$ Longitudinal velocity of the vehicle

2.3.6. Normal Force: The normal forces applied to the tires vary as a function to longitudinal and side accelerations of the vehicle. The expressions of these forces are given by [15]

$$
F_{N}=\frac{M s}{2 *\left(L_{1}+L_{2}\right)}(g * L 2-h * V x)
$$

$\dot{V}_{x}$ s the longitudinal acceleration of the vehicle

2.3.7. Steering-Wheel Angle: The Steering-Wheel angle as chosen as mentioned in the Figure 6. 


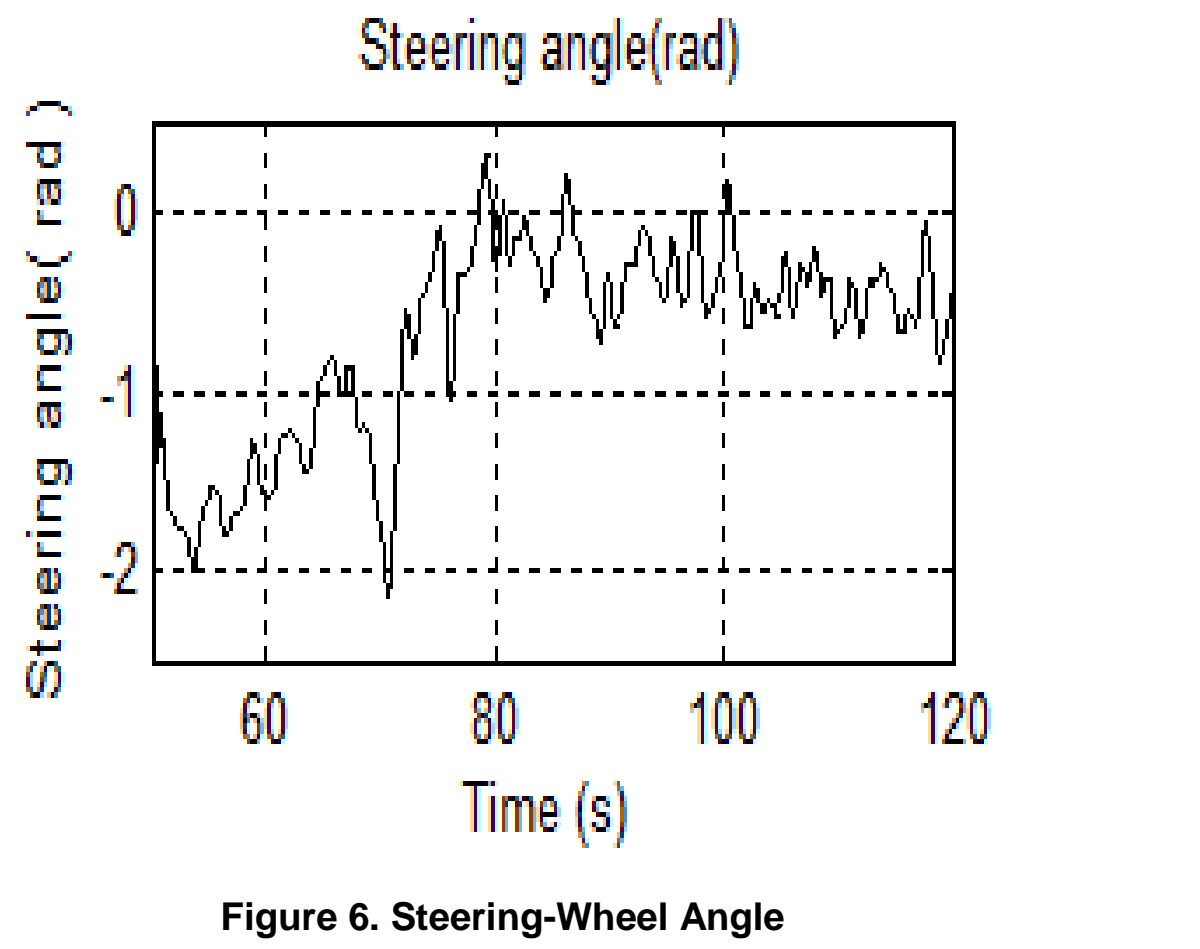

\section{Results and Analyzes}

In this part, we use real entered signals of the road provided by IFSTTAR for three types of lanes (dry, wet, glaze), the vehicle olls at a mean lelocity of $50 \mathrm{~km} / \mathrm{h}$.

In what follows we will represent the effect of the three tracks on the simulated dynamic behavior of the vehicle taking in account the orders of driver, the plan of the road represented by the angles of Eutier Rolling-Pitching-Yaw $\left(\psi_{x}, \psi_{y}, \psi_{z}\right)$ as illustrated previously and the forces of the contacts given by the formyla of Pacejeka[13].

\subsection{Effects on the Side and Congitudinal Slip}

Figure 7 illustrates the evolution of the sideslip angle of the left front steerable wheel and their longitudinal slip in tume for the three types of tracks.

It is note that, the sideslip angle of left front wheel follows the steering-wheel angle given by Figure 6, with a certain shift in the negative direction for the wet tracks, in the case of the two phases of steering. In the case of longitudinal slip, it is noted that the longitudinal slip almost reached $5 \%$ during the first phase of steering and then it returns to a value lower than $1 \%$ and there remains stable around this value. 

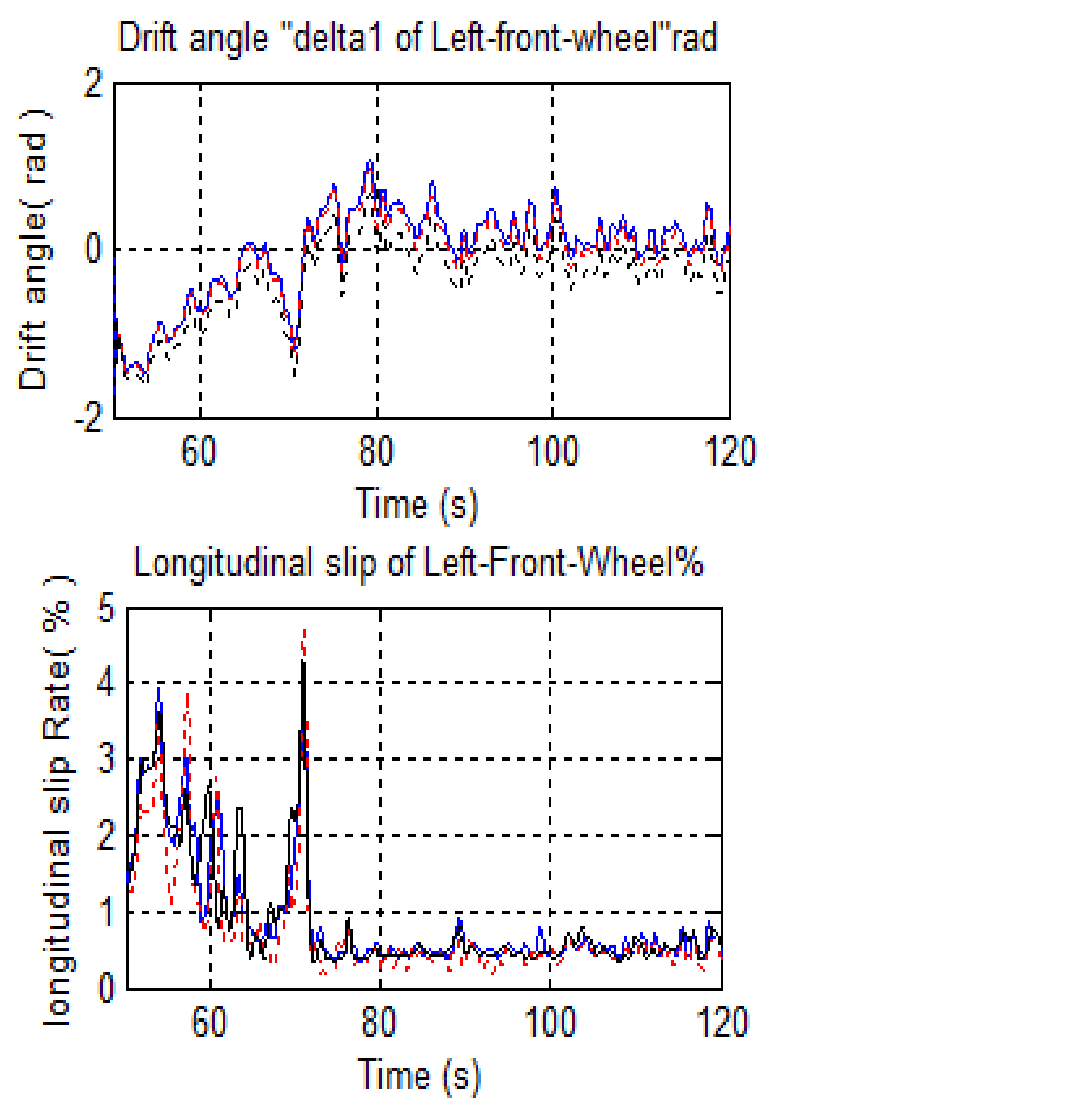

Figure 7. Effect Tracks to the Drift Angle and Longitudinal Slip Ratio (Bleu-dry

\subsection{Effects on the Wheels Velocities}

$$
\text { Lare, Red-wetlane and Black-glaze Lane) }
$$

We illustrated in figure 8 the case of left front steerable wheel to clear up the effect of the three tracks on linear and rotations velocities of the wheel.

It is well observed that the rotation velocity is around $52 \mathrm{rad} / \mathrm{s}$ in the case of the track dry and wet what translates that linear velocity is around $14 \mathrm{~m} / \mathrm{s}$. But in the case of the track glaze it is observed that speeds in extreme cases tend to be annulled. What explains why the vehicle starts to enter a total slip.

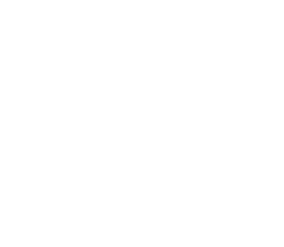


Longitudinal velocity of the left-front-wheel

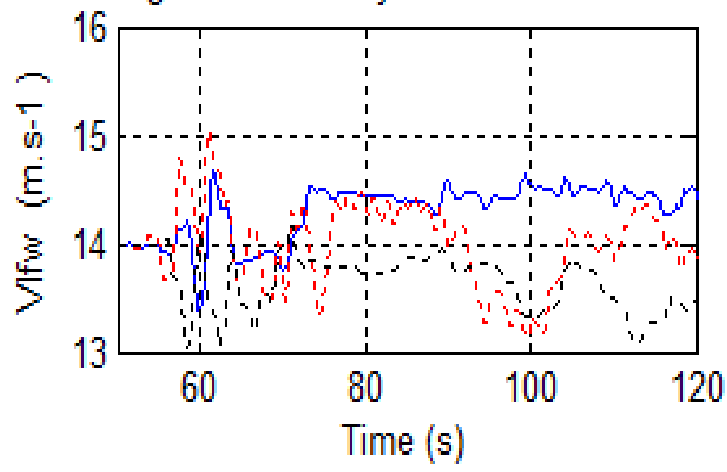

\section{Rotate velocity of the left front wheel (rad.s-1)}

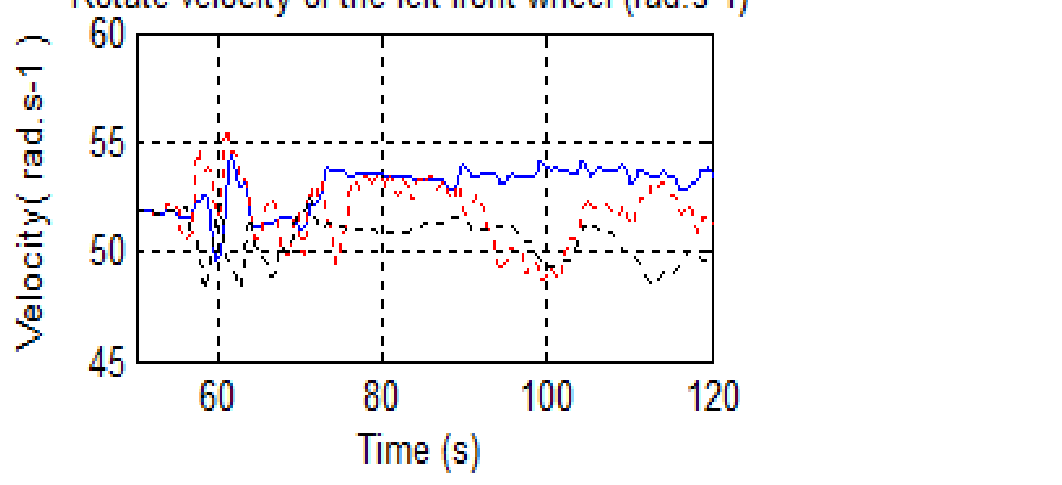

Figure 8. Effect Tracks on the Linear and Rotations Velocities of the Wheels (Bleu-dry Lane, Red-wet Lane and Black-glaze Lane)

\subsection{Effects on the Suspensions of the Four Wheels}

The effects of three tracks on the clearance acceleration at suspensions are given by Figure 9. It is noted that in the dry track case, the acceleration is proportional to the plan of road given by figure 5 on the other hand in the case of the wet track and glaze the acceleration of clearance in the course of time tends to increase this. This is translated by a fast increase in clearances of the suspensions. Indeed this increase influences directly the stability of the vehicle.

\subsection{Effects on the Chassis}

We represented here the effect of adherence on longitudinal, side and vertical acceleration of the chassis given by Figure 10 and one represented their effect on the angles of orientation of the chassis given by the angles rolling-pitching-yaw illustrated by Figure 11.

\section{It is notieed that:}

Longitudinal acceleration, decrease with each increase of the rate of humidity.

- The side and the vertical clearances acceleration increase with each increase of the rate of humidity.

One can say from some remarks that the vehicle with each increase in the rate of humidity in the course of time tends to slip into the longitudinal direction and to deviate laterally with more clearances vertically. 

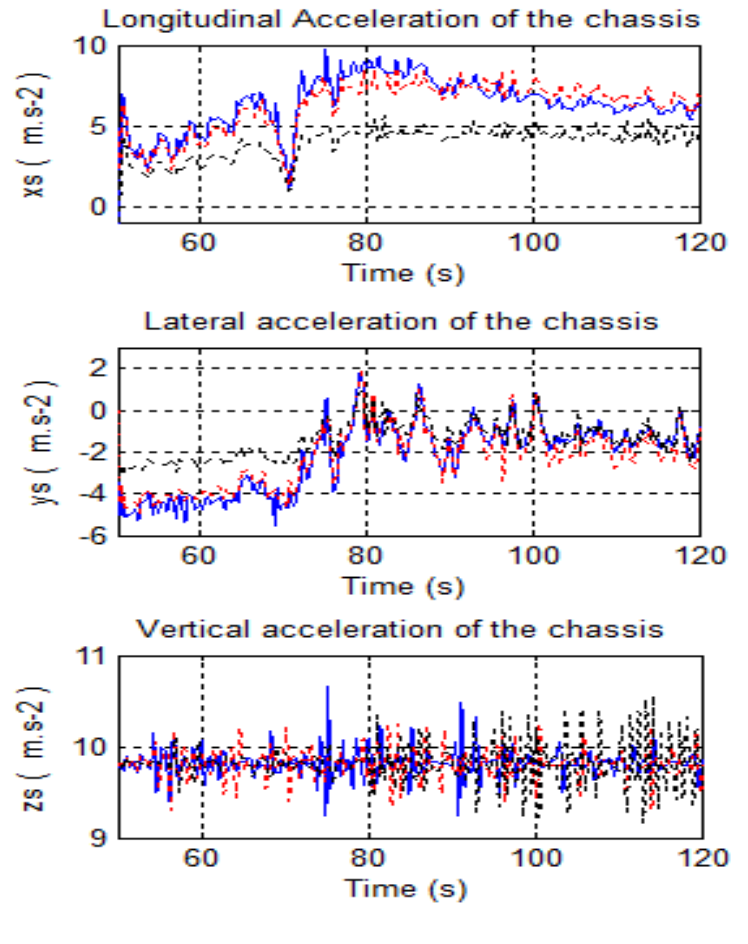

\section{Figure 10. Effect Tracks on the Acceleration Longitudinal, Lateral and Vertical (Bleu-dry Lane, Red-wet Lane and Black-glaze Lane)}

On Figure 11 one observes that the angles rolling-pitching-yaw tend to this diverged in time with each increase from the rate humidity.

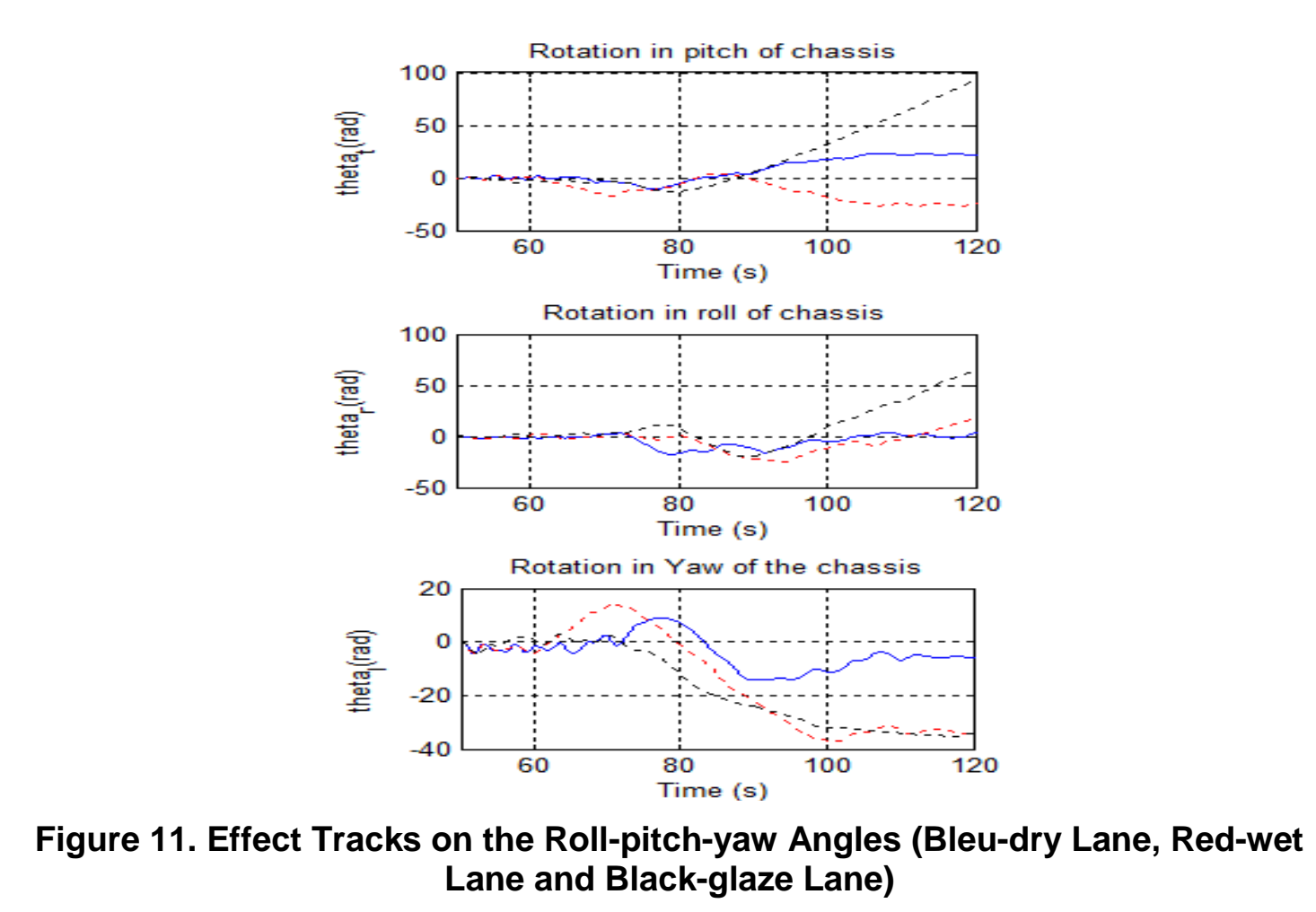




\subsection{Effects on the Generalized Forces Contacts of the Wheels}

The curves of evolution in the time of the generalized longitudinal, side and vertical forces in the space articulated according to the nature of track as given by Figure 12 .

It is noted that:

- The component resulting from the generalized longitudinal and vertical force decrease, with each increase for the rate humidity

- The component resulting from the generalized side force increases with each increase for the rate humidity.

Finally, we can note that the dynamic behavior of the vehicle depends absolutely on the nature of coating of the track on which the vehicle runs.
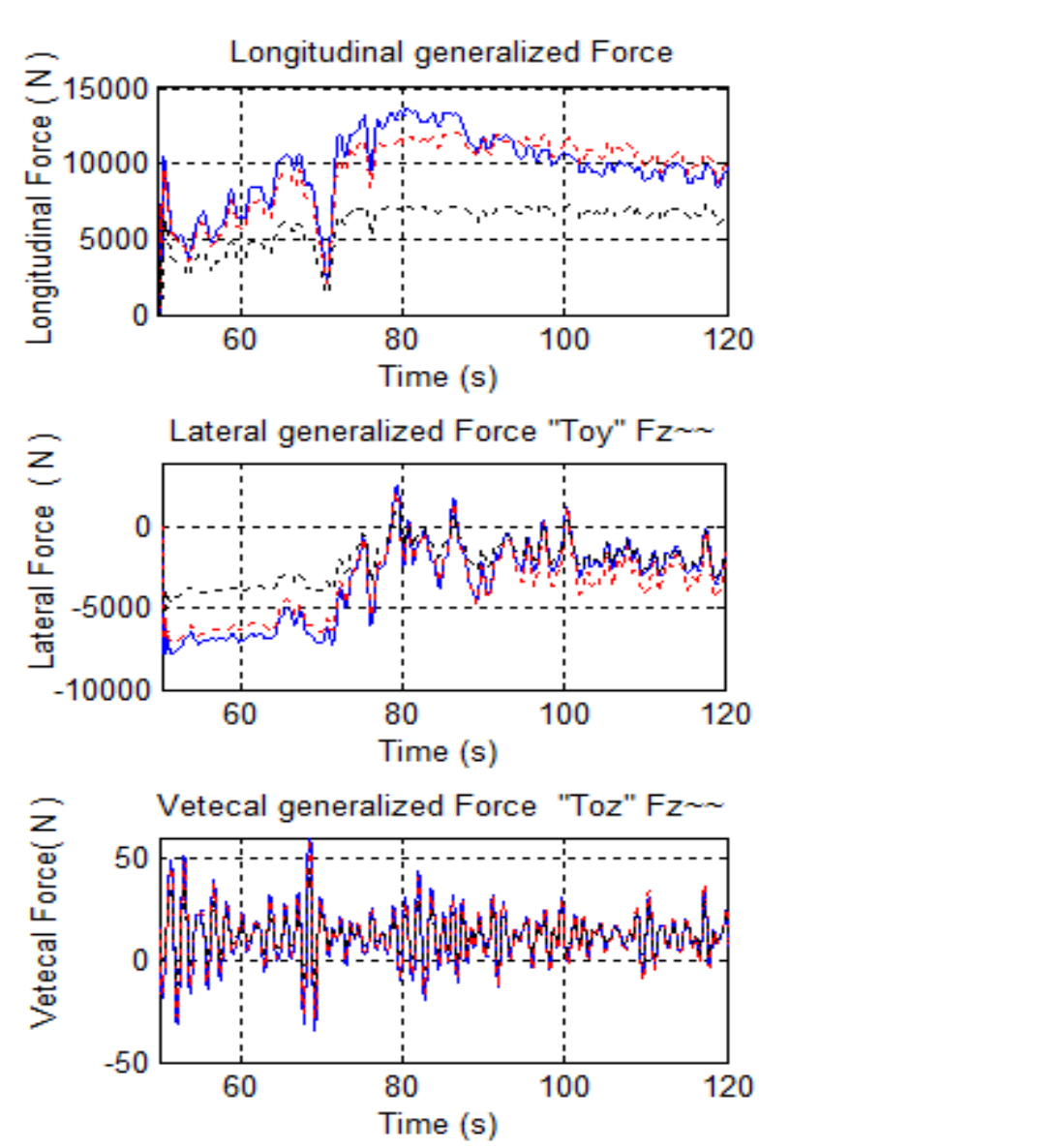

Figure 12. Effect Tracks on the Gneral Forces Longitudinal, Lateral and Vertical (Bleu-dry lane, Red-wet lane and Black-glaze Lane)

\section{Conclusion}

We proposed in this paper a simulator of a nominal model of the road vehicle rather near to the real physical system, which makes it possible to describe its dynamics. Consequently, we based on signals of input real of the road to describe the influence of adherence on the dynamic behavior of the vehicle. 
We represented like results of the effects of adherence according to water content's on the longitudinal, sideslip, and speed of the wheels, clearance of the suspensions, orientation of the frame and on the generalized forces of contacts wheels-ground.

This simulator can be wide while inserting new components to see their influence on the vehicle handling; it will be also useful for the design of laws of command for the control of the vehicle.

Appendix: Parameters of the models

* Inertia matrix of the system

$M(q)(16 \times 16)=$

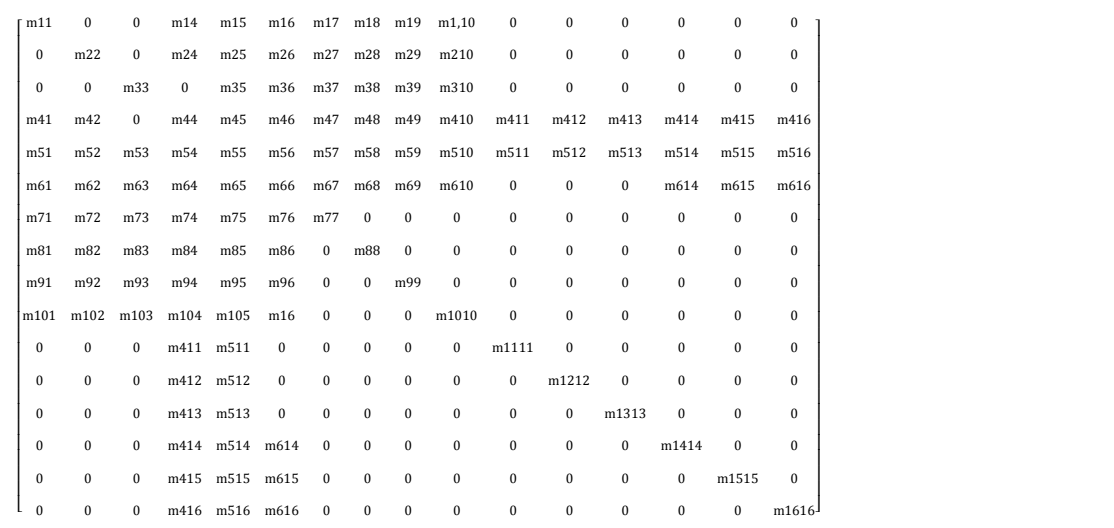

Coriolis and Centrifuges terms matrix

$\mathrm{C}(\mathrm{q}, \dot{q})(16 X 16)=$

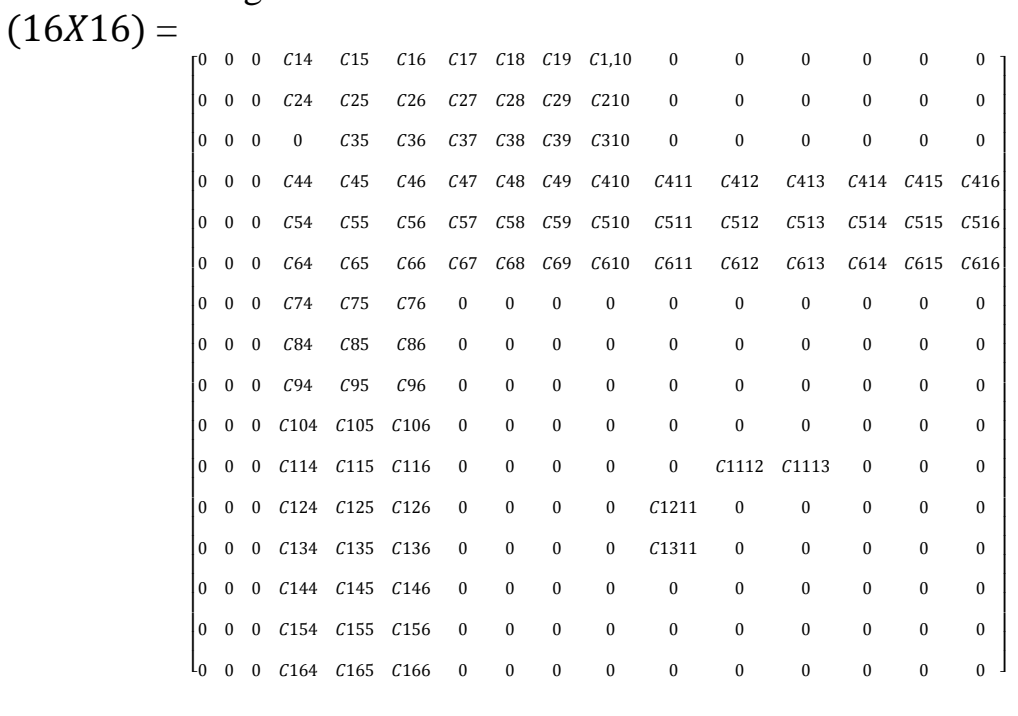

The form symbolic system of the jacobian matrix of the four points of contact (wheels) is:

${ }^{\mathrm{T}}(16 \times 12)=$ 


\section{References}

$$
\left[\begin{array}{cccccccccccc}
1 & 0 & 0 & 1 & 0 & 0 & 1 & 0 & 0 & 1 & 0 & 0 \\
0 & 1 & 0 & 0 & 1 & 0 & 0 & 1 & 0 & 0 & 1 & 0 \\
0 & 0 & 1 & 0 & 0 & 1 & 0 & 0 & 1 & 0 & 0 & 1 \\
\mathrm{~J} 41 & \mathrm{~J} 42 & 0 & \mathrm{~J} 44 & \mathrm{~J} 45 & 0 & \mathrm{~J} 47 & \mathrm{~J} 48 & 0 & \mathrm{~J} 410 & \mathrm{~J} 411 & 0 \\
\mathrm{~J} 51 & \mathrm{~J} 52 & \mathrm{~J} 53 & \mathrm{~J} 54 & \mathrm{~J} 55 & \mathrm{~J} 56 & \mathrm{~J} 57 & \mathrm{~J} 58 & \mathrm{~J} 59 & \mathrm{~J} 510 & \mathrm{~J} 511 & \mathrm{~J} 512 \\
\mathrm{~J} 61 & \mathrm{~J} 62 & \mathrm{~J} 63 & \mathrm{~J} 64 & \mathrm{~J} 65 & \mathrm{~J} 66 & \mathrm{~J} 67 & \mathrm{~J} 68 & \mathrm{~J} 69 & \mathrm{~J} 610 & \mathrm{~J} 611 & \mathrm{~J} 612 \\
\mathrm{~J} 71 & \mathrm{~J} 72 & \mathrm{~J} 73 & 0 & 0 & 0 & 0 & 0 & 0 & 0 & 0 & 0 \\
0 & 0 & 0 & \mathrm{~J} 84 & \mathrm{~J} 85 & \mathrm{~J} 86 & 0 & 0 & 0 & 0 & 0 & 0 \\
0 & 0 & 0 & 0 & 0 & 0 & \mathrm{~J} 97 & \mathrm{~J} 98 & \mathrm{~J} 99 & 0 & 0 & 0 \\
0 & 0 & 0 & 0 & 0 & 0 & 0 & 0 & 0 & \mathrm{~J} 1010 & \mathrm{~J} 1011 & \mathrm{~J} 1012 \\
0 & \mathrm{~J} 112 & 0 & 0 & 0 & 0 & 0 & 0 & 0 & 0 & 0 & 0 \\
0 & 0 & 0 & 0 & \mathrm{~J} 125 & 0 & 0 & 0 & 0 & 0 & 0 & 0 \\
\mathrm{~J} 131 & 0 & 0 & 0 & 0 & 0 & 0 & 0 & 0 & 0 & 0 & 0 \\
0 & 0 & 0 & \mathrm{~J} 144 & 0 & 0 & 0 & 0 & 0 & 0 & 0 & 0 \\
0 & 0 & 0 & 0 & 0 & 0 & \mathrm{~J} 157 & 0 & 0 & 0 & 0 & 0 \\
0 & 0 & 0 & 0 & 0 & 0 & 0 & 0 & 0 & \mathrm{~J} 1610 & 0 & 0
\end{array}\right]
$$

[1] J. Villagra, B. d'Andrea- Novel, M. Fliess and H. Mounter, A diagnostic-based approach for tire-road forces and maximum friction estimation", In Control Engineering Practicem, (2010).

[2] S. Muller, M. Uchanski and K. Hedrick, "Estimation of the Maximum Tire-Road Friction Coefficient", Journal of Dynamic Systems, Measurement, vol. 125 / 607, (2003),

$$
\text { S }
$$

[3] H. Fischein, R.Gnadier, H.Unrau, "The Influence of the Track Surface Structure on The Frictional Force Behaviour of Passenger Car Tyres in Dry and Wet Track Surface Conditions", ATZ, vol. 10, (2001), pp. 950962.

[4] H. Imine, L. Fridman, H. Shraim, and M. Djemai, "Sliding Mode Based Analysis and Identification of Vehicle Dynamics", Lecture Notes in Control and Information Sciences, Library of Congress Control Number: 2011932321, (2011); Springer-Verlag Berhin Heidelberg.

[5] J. J. Craig. Introduction to robotics. Mechanics and control. Addison- Wesley, (1989).

[6] W. Khalil and E DombreModelling, "Identification and control of robots", Hermès Penton, (2002), London \& Paris.

[7] A. El Hadhri, "Modélisation de véhicules, observation d'état et estimation des forces pneumatiques: Application au contrôle longitudinal", These de doctorat, Université de Versailles-Saint-Quentin en Yvelines, (2001).

[8] B. Zami, "Contribution à l'identification de la liaison Véhicule-Sol d'un véhicule automobile Estimation des paramètres de modèles depneumatiques", PhD thesis, Université de Haute-Alsace, (2005).

[9] M. Oudghiri "Commande multi modéles tolérante aux défauts :Application au controle de la dynamique d'un véhicule automobile", doctora de université de picardie jules verne, Université de picardie jules verne, (2008).

[10] O. Orfila, "Influence de 1 infrastructure routière sur l'occurrence des pertes de contrôle de véhicules légers en virage : modélisation et validation sur site expérimental", Thèse de doctorat, Université D’Evry-Val-d'Essone, (2009).

[11] S. Maakaroun, "Modélisation et simulation dynamique d'un véhicule urbain innovant en utilisant le formalisme de la robotique", Thèse de doctorat, Université de Nantes angers le mans, (2011).

[12] M. Ouani, "Observation de système à entrées inconnues, applications à la dynamique automobile", Thèse de doctorat, Université de Limoges, (2011).

[13] H.B Pacejka,, Besseling, Magic Formula Tyre Model with Transient Properties. 2nd International Tyre colloquium on Tyre Models for Vehicle Dynamic Analysis, (1997); Berlin, Germany.

[14] N. Hamadi, H. Imine et D.-E. Ameddah, "Outils Principaux Pour le Développement d'un Modèle de Véhicule articulé tout Terrain", International Conference on Syatems and Processing Information, (2013); Guelma, Algeria.

[15] N. K. M'Sirdi, B. Jaballah, A. Naamane and H. Messaoud, "Robust Observers and Unknown Input Observers for estimation, diagnosis and control of vehicle dynamics", IROS, (2008); NICE, France. 


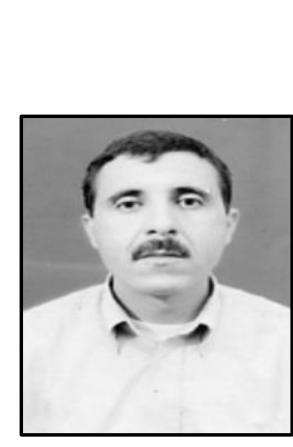

\begin{abstract}
Authors
Nacer Hamadi, received the Engineering degrees in Control for electronically engineering from the Batna University, Algeria, in 1995. Later, he has been working for 10 years as state engineer of the laboratories in the University of Constantine, Algeria and he has received the Magister in Robotics for electronically engineering at this period. Currently he has doctoral student in the field of robotics. His main research interests include dynamics and control of a robots and vehicle ground.
\end{abstract}

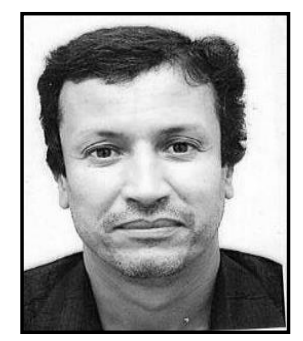

Djamel-eddine Ameddah, received the Masters degrees in electronics engineering from the National Polytechnic School of El-Harrach Algeria, in 1984. Holds his Ph.D in Electrical Engineering obtained from collaboration between Algerian and French Universities in 2004. He has been responsible of the undergraduate program in the engineering school for many years. He was involved or leading several research projects including the actual project: 'modeling and contro/ of wheeled mobile robots'. His research interests include robotics traction control systems, mechatronics.

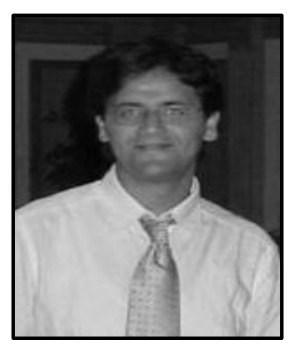

Hocine Imine, feceived the Master's degree and the Ph.D. degree in robotics and automation from Versailles University, Versailles, France, in 2000 and 2003 sespectively. From 2003 to 2004, he was a Researcher with the Robotic Laboratory of Versailles (LRV in French) and an Assistant Professor with Versailles University. In 2005, he joined mstitut Françals des Sciences et Technologies des Transports, where he is cumrently a Researcher. He received the Accreditation to Supervise Research (Habilitation à Diriger des Recherches-HDR) in March 2012. He is involved in different projects like véhicule interactil du futur (VIF) and heavy route (intelligent route guidance for heavy traffic). He is also responsible for the research project PLInfra on beavy vehicle safety and the assessment of their impacts on pavement and bridges. He has published two books, over 60 technical papers, and several industrial technical reports. His research interests include intelligent transportation systems, heavy vehicle modeling and stability, diagnosis, nonlinear observation, and nonlinear control. 
International Journal of Hybrid Information Technology

Vol.7, No.5 (2014)

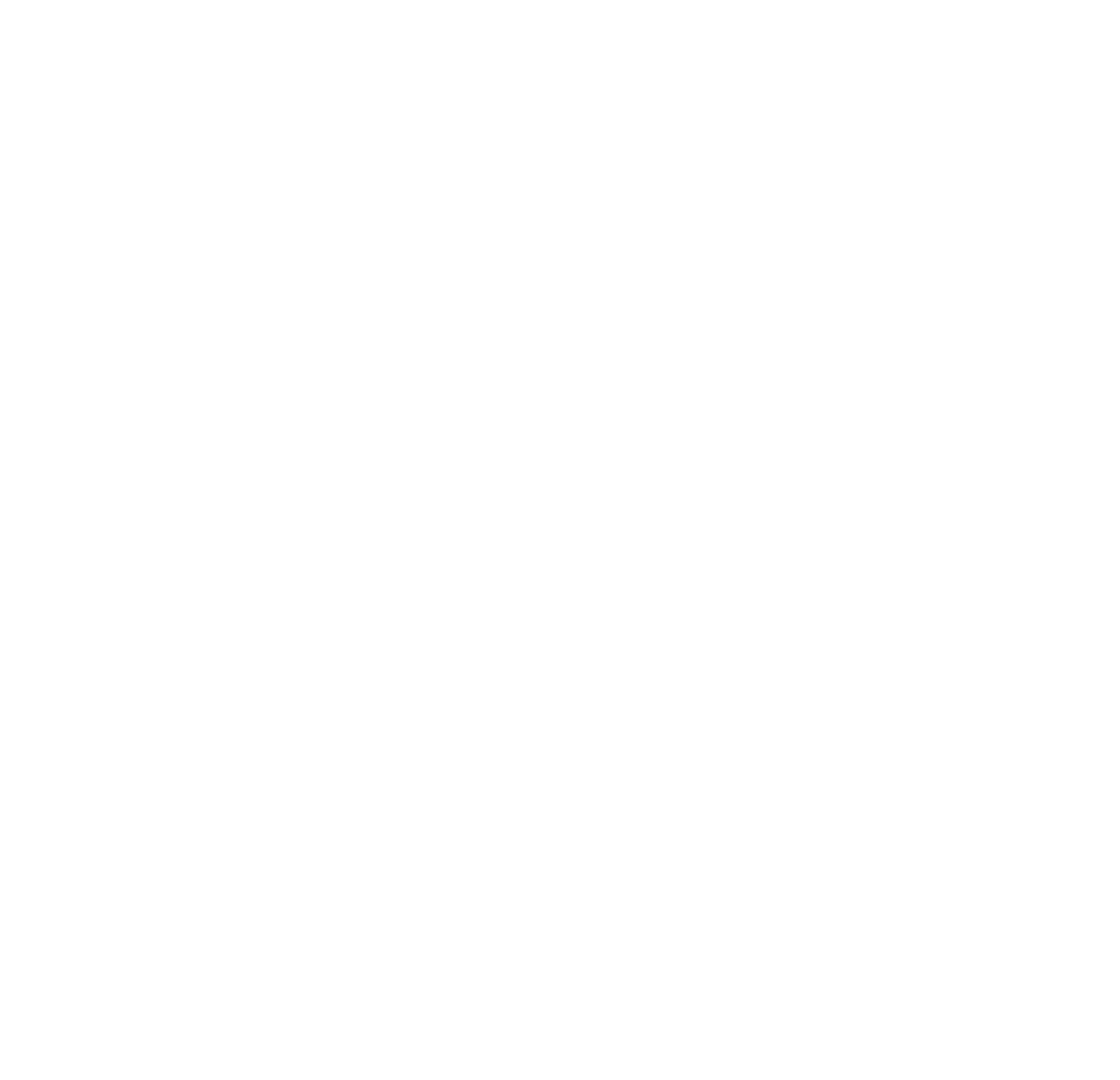

